\title{
Revealing the single electron pocket of FeSe in a single orthorhombic domain
}

\author{
Luke C. Rhodes $\odot,{ }^{1}$ Matthew D. Watson $\odot,{ }^{2}$ Amir A. Haghighirad $\odot,{ }^{3}$ D. V. Evtushinsky $\odot,{ }^{4}$ and Timur K. Kim $\oplus^{2,}{ }^{*}$ \\ ${ }^{1}$ School of Physics and Astronomy, University of St. Andrews, St. Andrews KY16 9SS, United Kingdom \\ ${ }^{2}$ Diamond Light Source, Harwell Campus, Didcot OX11 ODE, United Kingdom \\ ${ }^{3}$ Institute for Quantum Materials and Technologies, Karlsruhe Institite of Technology, 76021 Karlsruhe, Germany \\ ${ }^{4}$ Laboratory for Quantum Magnetism, Institute of Physics, École Polytechnique Fédérale de Lausanne, CH-1015 Lausanne, Switzerland
}

(Received 6 April 2020; accepted 13 May 2020; published 8 June 2020)

\begin{abstract}
We measure the electronic structure of FeSe from within individual orthorhombic domains. Enabled by an angle-resolved photoemission spectroscopy beamline with a highly focused beam spot (nano-ARPES), we identify clear stripelike orthorhombic domains in FeSe with a length scale of approximately 1-5 $\mu \mathrm{m}$. Our photoemission measurements of the Fermi surface and band structure within individual domains reveal a single electron pocket at the Brillouin zone corner. This result provides clear evidence for a one-electron-pocket electronic structure of FeSe, observed without the application of uniaxial strain, and calls for further theoretical insight into this unusual Fermi surface topology. Our results also showcase the potential of nano-ARPES for the study of correlated materials with local domain structures.
\end{abstract}

DOI: 10.1103/PhysRevB.101.235128

\section{INTRODUCTION}

Electronic nematicity, a correlated electronic state which breaks rotational symmetry, is a common phenomenon in many strongly correlated materials, including cuprates [1], heavy-fermion systems [2], and iron-based superconductors [3]. In the iron-based superconductors, nematicity manifests as a tetragonal-to-orthorhombic structural phase transition which, despite a very small change in lattice constants, coincides with large anisotropic responses in measurements that are sensitive to the material's electronic structure [4]. It has been postulated that this nematic state may be directly related to the superconducting properties of these materials [5], however, because of the proximity of the nematic phase to stripelike antiferromagnetic ordering in most compounds, determining the key properties of a pure nematic state has proved challenging.

FeSe is an ideal system for investigating the origin of the nematic state, as it exhibits a well-defined nematic transition at $T_{s}=90 \mathrm{~K}$ without the presence of long-range magnetic order [6-8]. The electronic structure of FeSe has been extensively investigated by angle-resolved photoemission spectroscopy (ARPES) and significant changes in the band structure can be observed to onset at the nematic transition [9-14]. However, the typical size of the beam spot used for high-resolution synchrotron ARPES measurements is of the order of $50 \mu \mathrm{m}$ or larger [15], while individual orthorhombic domains can be of the order of only $1-10 \mu \mathrm{m}$ [16]. Therefore conventional ARPES measurements will usually

\footnotetext{
*timur.kim@diamond.ac.uk
}

Published by the American Physical Society under the terms of the Creative Commons Attribution 4.0 International license. Further distribution of this work must maintain attribution to the author(s) and the published article's title, journal citation, and DOI. detect a superposition from both domains with approximately equal intensity. Several groups have attempted to overcome this limitation by aligning the orthorhombic domains along a preferential axis by applying uniaxial strain [17-20], commonly known as "detwinning." These measurements revealed a single elliptical holelike pocket at the Brillouin zone center, in line with theoretical predictions [21]. However, they also revealed the presence of only a single electronlike pocket around the $\mathrm{M}$ and $\mathrm{A}$ points at the Brillouin zone corner, despite the expectation of two electron pockets in most theoretical models $[11,13,21,22]$. The theoretical understanding of this discrepancy remains controversial and unresolved [18,23-27].

There is, however, a more favorable approach to study the electronic structure of FeSe. Rather than applying uniaxial strain to the sample, which may alter the material's physical properties or deform its surface, one can focus a photon beam spot below the typical length scale of the domains. This constitutes a significant technical challenge, requiring the combination of good spatial, angular, and energy resolutions, with a photon energy and geometry set to reach the more intriguing bands near the Brillouin-zone corner, while also maintaining a reasonable count rate and keeping the sample position and temperature under control. However, recent progress in nano-ARPES makes such experiments achievable $[28,29]$, and here we report the first such measurements of the nematic state of FeSe.

In this paper, we use the highly focused nano-ARPES beam spot to measure the electronic structure of FeSe domain by domain. We begin by detecting regular stripelike orthorhombic domains with periods of $1-5 \mu \mathrm{m}$. By measuring the electronic structure from within a single domain, we reveal that the states around the $\mathrm{M}$ point are consistent with the one-electron-pocket description observed in ARPES measurements on detwinned crystals. This result provides direct and apparent evidence for a one-electron pocket at the $\mathrm{M}$ point in 
FeSe and calls for deeper theoretical insight into the origin of the electronic nematic state. Our measurements showcase the potential for measuring single-domain electronic structures in correlated materials using the technique of nano-ARPES.

\section{METHODS}

Single crystals were grown using the chemical vapor transport method $[10,30]$. Nano-ARPES measurements were performed at the I05 beamline at the Diamond Light Source, Oxfordshire, U.K. [15]. Fresnel-zone plate technology is used to focus the beam. While the beamline is capable of spatial resolutions better than $\sim 500 \mathrm{~nm}[28,31]$, our measurements are performed with a beam spot (full width at half-maximum) closer to $1 \mu \mathrm{m}$, as a compromise to achieve a higher count rate. The beamline is equipped with a Scienta DA30 analyzer. [32]. We use a photon energy of $58 \mathrm{eV}$ in linear vertical polarization, chosen to access the A point, where the electron bands are slightly better separated than at M [11]. The total energy resolution was approximately $30 \mathrm{meV}$ and the angular resolution was $0.3^{\circ}$. Thus, the energy resolution is not comparable to that of the highest-resolution ARPES measurements on FeSe [11,23,33], as some compromise is necessary to achieve a reasonable count rate; nevertheless, it is sufficient to resolve the bands with clarity. The sample temperature was set at $43 \mathrm{~K}$ throughout the experiment, except for the measurements of the tetragonal state [Figs. 2(d)-2(f)] at $115 \mathrm{~K}$.

\section{RESULTS}

In order to establish the presence of a domain structure, we must begin by analyzing spatial mapping data. As shown schematically in Fig. 1(a) it is necessary that our beam spot must be smaller than the typical domain spacing. We choose the experimental geometry as sketched in Fig. 1(b), where, according to the one-electron-pocket scenario, only the "red" domain should show an electron pocket at the Fermi level. In Fig. 1(c) we show a spatial map of the surface of FeSe, where the color map of each pixel corresponds to the total number of photoelectrons in the detector. This hints at some underlying domain structure, but the variation in total intensity is only of the order of $10 \%$; in other words, the two domain orientations give a similar but not identical number of total photoelectrons in this measurement geometry. However, the true domain structure can be much more clearly revealed by integrating the counts detected within a particular region of interest (ROI) in momentum and energy space. We present this map in Fig. 1(d), where we have used two polygon-shaped ROIs defined by Fig. 1(e). Using a divergent red-blue color scale for emphasis, we can identify multiple stripelike domains, with a typical length scale of 1-5 $\mu \mathrm{m}$. In our geometry, the spatial $x$ (a)

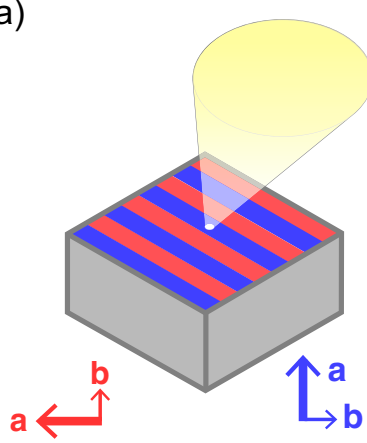

(b)

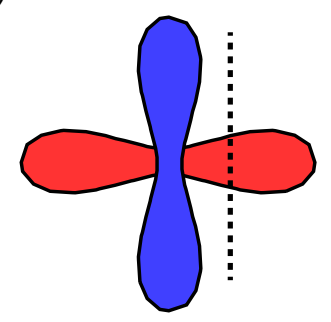

(c)
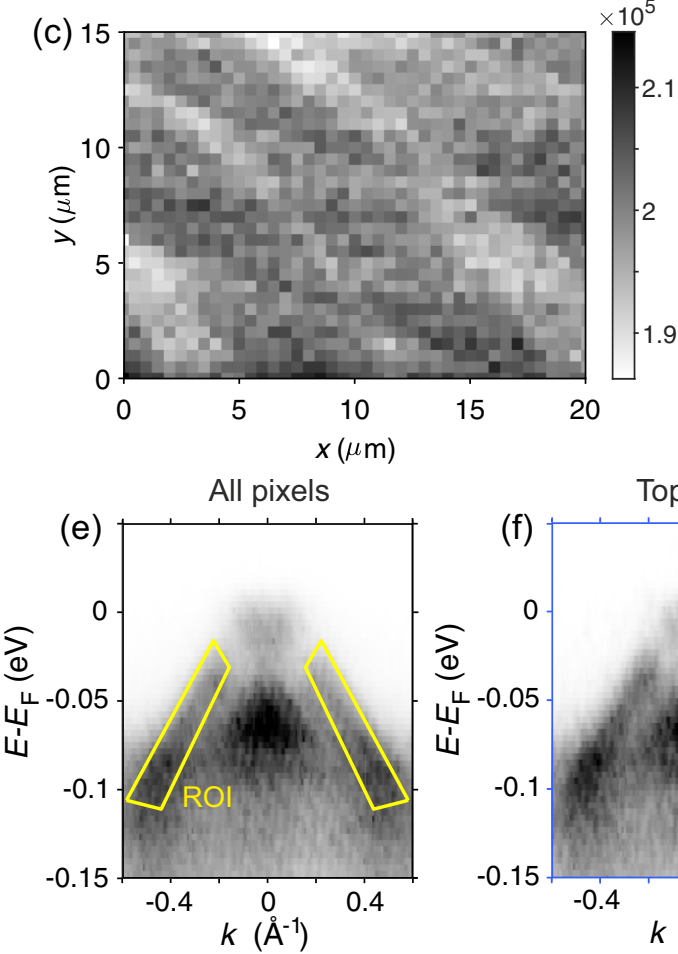

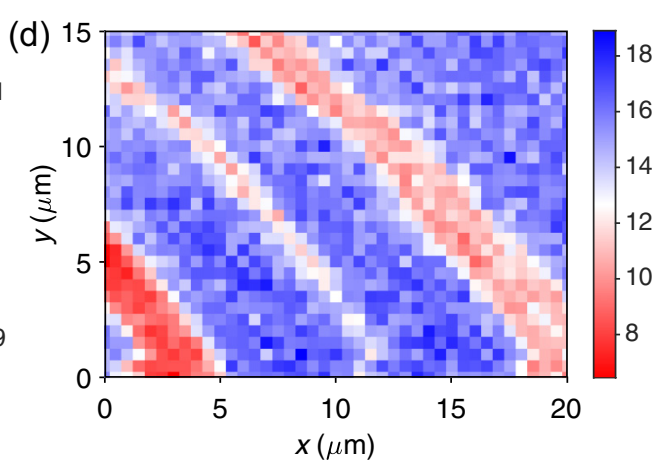

Top $12 \%$

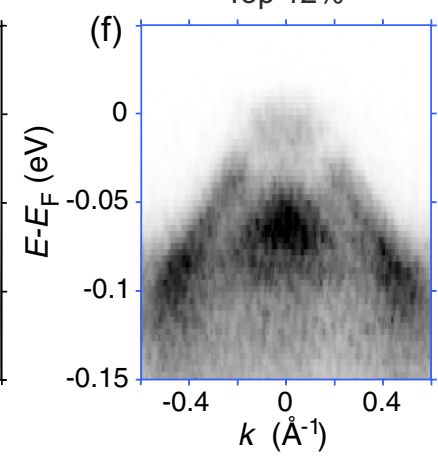

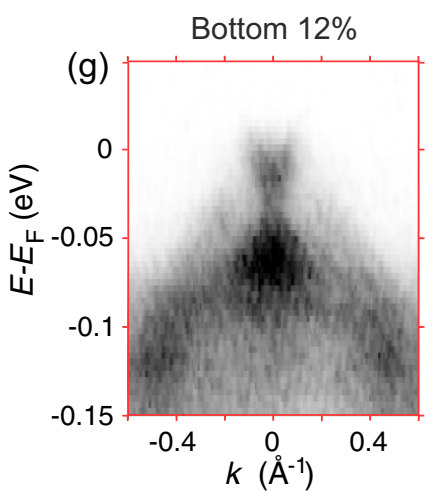

FIG. 1. Domain structure of FeSe. (a) Schematic of the experiment. Red and blue stripes represent domains with $90^{\circ}$-rotated orthorhombic axes. Nano-ARPES measurements focus the photon beam to around $1 \mu \mathrm{m}^{2}$, which allows the detection of photoelectrons from a single domain only. (b) Sketch of the cut in momentum space, just off the high-symmetry M point, used here to uncover the orthorhombic domain structure. In a one-electron-pocket scenario, the band dispersions along this cut will include an electron band crossing the Fermi level in one domain only. (c) Total photoelectron intensity real-space map of a region of cleaved surface of FeSe, taken at $h v=58 \mathrm{eV}$ in 0.5 - $\mu \mathrm{m}$ steps. (d) The same map as in (c) but with the photoelectron intensity integrated only within the region of interest (ROI) shown in (e). (e) Energy distribution map obtained by the summation of all pixels. The ROIs used in (d) are highlighted in yellow. (f, g) Energy distribution maps obtained by summing the top $12 \%$ of pixels from (d) (i.e., from blue areas) and the bottom $12 \%$ of pixels from (d) (from red areas) . 
and $y$ axes correspond approximately to the Fe-Fe bond directions, i.e., the domain boundaries are at $45^{\circ}$ to the $\mathrm{Fe}-\mathrm{Fe}$ directions, in keeping with previous results on other iron-based superconductors $[4,16]$. Interestingly, the "blue" domain appears to be preferential within this $20 \times 15 \mu \mathrm{m}$ window, which suggests that there may be a small local strain in this area of the sample which favors this orientation over the other.

To clarify that we are sensitive to the different band dispersions in the two domains, we perform a filtering procedure on this four-dimensional mapping data to take the spatial pixels which contain either the top $12 \%$ or bottom $12 \%$ intensity within the ROI and sum over these pixels to produce the energy distribution maps in Figs. 1(f) and 1(g). The top $12 \%$ of pixels from Fig. 1(d), corresponding to the blue domains, shows intense holelike bands extending towards, but not reaching, the Fermi level. In contrast, the bottom $12 \%$, corresponding to the red domains, reveals a small bright electron pocket, in accordance with the expectation of only one domain giving a Fermi-crossing band in this geometry [34].

Now that we have established the domain structure of our sample, we can study the band dispersions through the highsymmetry A point. To do this, we have taken $45^{\circ}$ line scans, i.e., equal steps in $x$ and $y$, bisecting the orthorhombic domains shown in Fig. 1(d). The intensity measured around the Fermi level is presented in Fig. 2(a). In the red domains, the bright and centralized intensity is consistent with a single band with a small $k_{F}$ of $\sim 0.05 \AA^{-1}$, which is consistent with the $k_{F}$ value of the minor length of the electron pocket determined by high-resolution ARPES [11]. In contrast, the blue domain shows less intensity within the $\sim 0.1 \AA^{-1}$ region (there is still a holelike band present near $E_{F}$ so there is some central intensity) but, instead, presents a sharp feature at a larger $k_{F}$ value of $\sim 0.2 \AA^{-1}$, again consistent with published values for the $k_{F}$ value of the longer axis of the electron pocket at $\mathrm{A}$. By averaging over a few points of the line scan that are well within individual domains, as indicated in Fig. 2(a), we achieve the effective single-domain high-symmetry energy distribution maps presented in Figs. 2(b) and 2(c). In Fig. 2(b) a small electron pocket can be observed with a minimum near $E_{F}$, and a large dispersion broadly centered around $-50 \mathrm{meV}$, whereas in Fig. 2(c) a large electronlike band dispersion can be observed, which exhibits a minimum around $-50 \mathrm{meV}$, and an additional holelike dispersion, which extends towards, but does not reach $E_{F}$. In both dispersions, a broad band centered just below $-50 \mathrm{meV}$ can be detected. Overall, the spectra closely resemble ARPES measurements on detwinned crystals [17].

At $115 \mathrm{~K}$, above the nematic transition, tetragonal symmetry is restored. Thus, in Fig. 2(d) the $k_{F}$ values as a function of the spatial distance now appear constant, and in Figs. 2(e) and 2(f) we observe identical band dispersions, indicating the absence of any significant spatial variation. These spectra are in agreement with dispersions observed at the A point in the tetragonal phase $[11,35]$ and indicate the presence of two electron pockets above $T_{s}$.

Finally, we present Fermi surface maps and high-statistics dispersions obtained from static positions, precisely chosen to be well within each of the domains. The Fermi surface maps around the A point [Figs. 3(b) and 3(e)] are obtained using the DA30 analyzer's deflector-mode mapping, while the sample position remains fixed. In either case, a single pocket
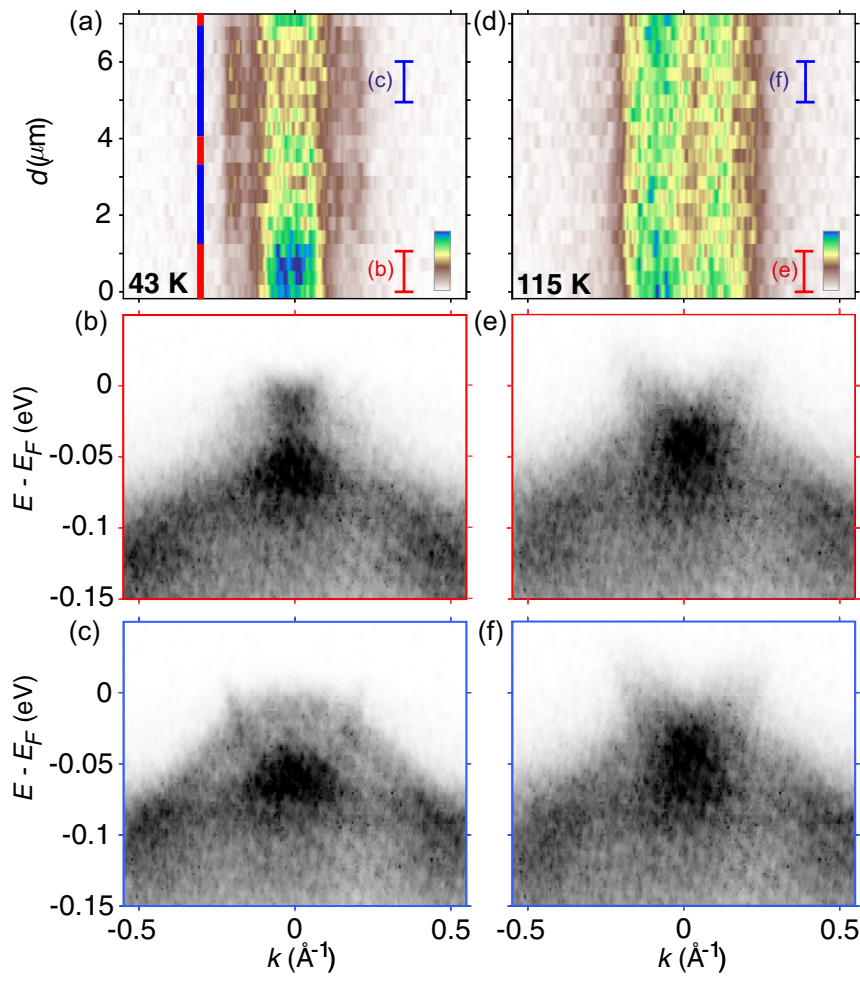

FIG. 2. Line scan across the domains. (a) Photoemission intensity integrated within $10 \mathrm{meV}$ of $E_{F}$ as a function of the spatial distance along a spatial cut perpendicular to the domains in Fig. 1(d). In $k$ space, the cut corresponds to the $\mathrm{A}-\mathrm{Z}$ direction. The red and blue striped bar is a guide to indicate the different orthorhombic domains. (b, c) Momentum distribution maps averaged over the area defined by the red and blue profiles in (a). (d-f) Equivalent measurements at $T=115 \mathrm{~K}$, where the lack of spatial dependence highlights the loss of any domain structure above $T_{s}$.

can be observed at the Fermi level, elongated along either the $k_{X}$ or the $k_{Y}$ direction, and is drawn schematically in Figs. 3(a) and 3(d). We further present high-statistics measurements in Figs. 3(c) and 3(f), obtained at the same spatial positions, which extend the electronic structure measurements from the A point towards the $\mathrm{Z}$ point of the second Brillouin zone. It can be seen that there is also some observable difference here: a larger $k_{F}$ value of the hole pocket is observed in Fig. 3(c) compared to Fig. 3(f), confirming that the elongation of the hole pocket is perpendicular to that of the electron pocket $[17,36]$. Overall, the dispersions closely resemble previous photoemission measurements on detwinned crystals of FeSe [17-20], though it should be noted that the geometry here is not fully equivalent to that in some reports [37]. We do not comment on the orbital characters of the bands, which have been strongly disputed $[11,20,25]$, but only note that the connectivity of bands from the $\mathrm{Z}$ to the A point (or $\Gamma$ to $\mathrm{M}$ ) is not obvious in such measurements due to the weak and broad features in between.

\section{DISCUSSION}

Our results are informative for understanding previous claims that a sample may be "accidentally detwinned" (e.g., 


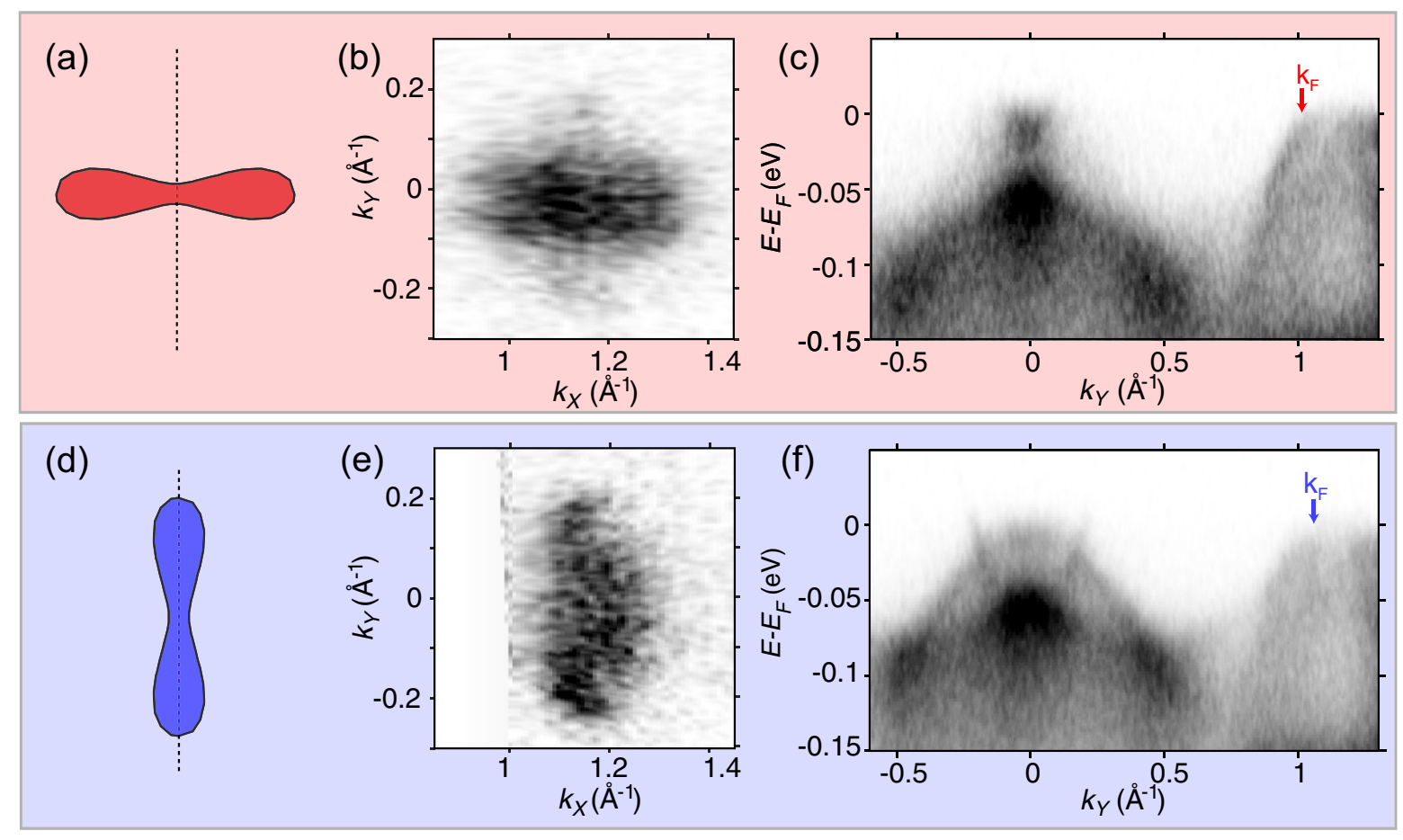

FIG. 3. Fermi surface around the A point and A-Z band dispersion from two orthorhombic domains. (a) Schematic of the single-electronpocket Fermi surface at the A point. (b) Measured Fermi surface map highlighting a single electron pocket elongated along the experimental $k_{X}$ axis. (c) A-Z band dispersion along the experimental $k_{Y}$ axis, as shown by the dashed line in (a). (d-f) Equivalent measurements from an adjacent domain with $90^{\circ}$-rotated orthorhombic axes. Here we use the notation $k_{X}$ and $k_{Y}$ to refer to the momentum with respect to the fixed experimental geometry $[17,18]$, where the analyzer angle corresponds to $k_{Y}$.

$[33,38])$. Our data show that, with a sufficiently focused light source, it may indeed be possible to "accidentally" land in uniform single-domain patches as large as $\sim 5 \times 5 \mu \mathrm{m}$ on an unstrained sample. However, it is more probable to find patches which exhibit a preferential orientation towards one domain, such as the dominance of the blue domain in Fig. 1(d), but do not purely consist of a single domain. Such variation on a more macroscopic length scale was observed in the laser micro-ARPES measurements by Schwier et al. [39] but was understood to correspond to local strain variation favoring particular domain orientations, rather than individual domains. The length scales we observe correspond to those obtained by polarized light imaging [16], but we caution that the sizes of domains may depend on many factors (sample quality and preparation, temperature, cooling rate, etc). We therefore propose that careful analysis of spatial mapping data, such as presented here, is the preferred method to justify any claim of obtaining ARPES spectra from a "single domain."

Within the context of the debate on the electronic structure of FeSe, our nano-ARPES results are consistent with those obtained by detwinning the sample, and we were unable to find any quantitative difference in band positions. This provides direct evidence that uniaxial strain has a negligible effect on the material's intrinsic electronic structure, at least up to the point where the sample is almost fully detwinned. Thus, the four detwinned ARPES measurements [17-20] along with this nano-ARPES experiment should solidify the one-electron-pocket Fermi surface of FeSe as the true nematic ground state of this material.
Such a dramatic shift between a two-electron-pocket A point at $100 \mathrm{~K}$ to a one-electron-pocket $\mathrm{A}$ point at low temperatures provides a natural explanation for many of the anisotropic properties detected in FeSe at low temperatures, including the resistivity anisotropy [16], strongly anisotropic spin susceptibility revealed by inelastic neutron scattering [40], and unusual twofold-symmetric momentum dependence of the superconducting gap $[23,41]$. It has also been shown that such an electronic structure is fully consistent with quasiparticle interference measurements [42] and seems consistent with the measured Sommerfeld coefficient of FeSe [10,43]. Despite the mounting experimental evidence, however, it remains challenging to identify a reasonable theoretical model of the electronic structure of FeSe which can describe the evolution of the Fermi surface from a two-electron-pocket tetragonal state to a one-electron-pocket nematic state. Discovering such a model would be a key step forward in understanding the origin and importance of nematicity within FeSe and potentially all iron-based superconductors. Recent theoretical calculations have suggested that hybridization between the $d_{x z}$ and the $d_{x y}$ states may be an important factor $[25,44]$.

In a wider context, nano-ARPES is a novel and fastimproving technique that has already had a significant impact in the field of two-dimensional materials, especially in heterostructures and devices [45-47]. However, thus far it has seen relatively few applications in traditional correlated electron systems [28,29], at least partially because the lower energy scales involved require a high energy resolution, which is challenging to combine with a micron-scale spatial resolution. Nevertheless, in this work we were able 
to measure nematic domains in $\mathrm{FeSe}$, where the length scale can be as short as $1-2 \mu \mathrm{m}$ and the energy scales are on the order of $20 \mathrm{meV}$. Since the formation of magnetic or structural domains is common in correlated systems, our results highlight the tremendous opportunities for the use of nano-ARPES to reveal new structures in correlated electronic phases.

\section{ACKNOWLEDGMENTS}

We thank C. Cacho for technical support during the experiment. We acknowledge Diamond Light Source for time on beamline I05-ARPES under Proposal SI23890. L.C.R. acknowledges funding from the Royal Commission for the Exhibition of 1851.

L.C.R. and M.D.W. contributed equally to this article.
[1] R. Daou, J. Chang, D. LeBoeuf, O. Cyr-Choiniére, F. Laiberté, N. Doiron-Leyraud, R. Liang, D. A. Bonn, W. N. Hardy, and L. Taillefer, Broken rotational symmetry in the pseudogap phase of a high-Tc superconductor, Nature 463, 519 (2010).

[2] R. Okazaki, T. Shibauchi, H. J. Shi, Y. Haga, T. D. Matsuda, E. Yamamoto, Y. Onuki, H. Ikeda, and Y. Matsuda, Rotational symmetry breaking in the hidden-order phase of $\mathrm{URu}_{2} \mathrm{Si}_{2}$, Science 331, 439 (2011).

[3] S. Kasahara, H. J. Shi, K. Hashimoto, S. Tonegawa, Y. Mizukami, T. Shibauchi, K. Sugimoto, T. Fukuda, T. Terashima, A. H. Nevidomskyy, and Y. Matsuda, Electronic nematicity above the structural and superconducting transition in $\mathrm{BaFe}_{2}\left(\mathrm{As}_{1-x} \mathrm{P}_{x}\right)_{2}$, Nature 486, 382 (2012).

[4] I. R. Fisher, L Degiorgi, and Z. X. Shen, In-plane electronic anisotropy of underdoped '122' Fe-arsenide superconductors revealed by measurements of detwinned single crystals, Rep. Prog. Phys. 74, 124506 (2011).

[5] R. M. Fernandes, A. V. Chubukov, and J. Schmalian, What drives nematic order in iron-based superconductors? Nat. Phys. 10, 97 (2014).

[6] Yu. V. Pustovit and A. A. Kordyuk, Metamorphoses of electronic structure of FeSe-based superconductors, Low Temp. Phys. 42, 995 (2016).

[7] A. I. Coldea and M. D. Watson, The key ingredients of the electronic structure of FeSe, Annu. Rev. Condens. Matter Phys. 9, 125 (2018).

[8] A. E. Böhmer and A. Kreisel, Nematicity, magnetism and superconductivity in FeSe, J. Phys.: Condens. Matter 30, 023001 (2017).

[9] K. Nakayama, Y. Miyata, G. N. Phan, T. Sato, Y. Tanabe, T. Urata, K. Tanigaki, and T. Takahashi, Reconstruction of Band Structure Induced by Electronic Nematicity in an FeSe Superconductor, Phys. Rev. Lett. 113, 237001 (2014).

[10] M. D. Watson, T. K. Kim, A. A. Haghighirad, N. R. Davies, A. McCollam, A. Narayanan, S. F. Blake, Y. L. Chen, S. Ghannadzadeh, A. J. Schofield, M. Hoesch, C. Meingast, T. Wolf, and A. I. Coldea, Emergence of the nematic electronic state in FeSe, Phys. Rev. B 91, 155106 (2015).

[11] M. D. Watson, T. K. Kim, L. C. Rhodes, M. Eschrig, M. Hoesch, A. A. Haghighirad, and A. I. Coldea, Evidence for unidirectional nematic bond ordering in FeSe, Phys. Rev. B 94, 201107(R) (2016).

[12] A. Fedorov, A. Yaresko, T. K. Kim, E. Kushnirenko, E. Haubold, T. Wolf, M. Hoesch, A. Grueneis, B. Buechner, and S. V. Borisenko, Effect of nematic ordering on electronic structure of FeSe, Sci. Rep. 6, 36834 (2016).

[13] L. Fanfarillo, J. Mansart, P. Toulemonde, H. Cercellier, P. Le Fèvre, F. Bertran, B. Valenzuela, L. Benfatto, and V. Brouet, Orbital-dependent Fermi surface shrinking as a fin- gerprint of nematicity in FeSe, Phys. Rev. B 94, 155138 (2016).

[14] M. D. Watson, A. A. Haghighirad, H. Takita, W. Mansur, H. Iwasawa, E. F. Schwier, A. Ino, and M. Hoesch, Shifts and splittings of the hole bands in the nematic phase of FeSe, J. Phys. Soc. Jpn. 86, 053703 (2017).

[15] M. Hoesch, T. K. Kim, P. Dudin, H. Wang, S. Scott, P. Harris, S. Patel, M. Matthews, D. Hawkins, S. G. Alcock, T. Richter, J. J. Mudd, M. Basham, L. Pratt, P. Leicester, E. C. Longhi, A. Tamai, and F. Baumberger, A facility for the analysis of the electronic structures of solids and their surfaces by synchrotron radiation photoelectron spectroscopy, Rev. Sci. Instrum. 88, 013106 (2017).

[16] M. A. Tanatar, A. E. Böhmer, E. I. Timmons, M. Schütt, G. Drachuck, V. Taufour, K. Kothapalli, A. Kreyssig, S. L. Bud'ko, P. C. Canfield, R. M. Fernandes, and R. Prozorov, Origin of the Resistivity Anisotropy in the Nematic Phase of FeSe, Phys. Rev. Lett. 117, 127001 (2016).

[17] M. D. Watson, A. A. Haghighirad, L. C. Rhodes, M. Hoesch, and T. K. Kim, Electronic anisotropies revealed by detwinned angle-resolved photo-emission spectroscopy measurements of FeSe, New J. Phys. 19, 103021 (2017).

[18] M. Yi, H. Pfau, Y. Zhang, Y. He, H. Wu, T. Chen, Z. R. Ye, M. Hashimoto, R. Yu, Q. Si, D.-H. Lee, P. Dai, Z.-X. Shen, D. H. Lu, and R. J. Birgeneau, Nematic Energy Scale and the Missing Electron Pocket in FeSe, Phys. Rev. X 9, 041049 (2019).

[19] S. S. Huh, J. J. Seo, B. S. Kim, S. H. Cho, J. K. Jung, S. Kim, Y. Y. Koh, C. I. Kwon, J. S. Kim, W. S. Kyung, J. D. Denlinger, Y. H. Kim, B. N. Chae, N. D. Kim, Y. K. Kim, and C. Kim, Absence of Y-pocket in 1-Fe Brillouin zone and reversed orbital occupation imbalance in FeSe, Commun. Phys. 3, 52 (2020).

[20] H. Pfau, S. D. Chen, M. Yi, M. Hashimoto, C. R. Rotundu, J. C. Palmstrom, T. Chen, P.-C. Dai, J. Straquadine, A. Hristov, R. J. Birgeneau, I. R. Fisher, D. Lu, and Z.-X. Shen, Momentum Dependence of the Nematic Order Parameter in Iron-Based Superconductors, Phys. Rev. Lett. 123, 066402 (2019).

[21] S. Mukherjee, A. Kreisel, P. J. Hirschfeld, and B. M. Andersen, Model of Electronic Structure and Superconductivity in Orbitally Ordered FeSe, Phys. Rev. Lett. 115, 026402 (2015).

[22] J. Kang, R. M. Fernandes, and A. Chubukov, Superconductivity in FeSe: The Role of Nematic Order, Phys. Rev. Lett. 120, 267001 (2018).

[23] L. C. Rhodes, M. D. Watson, A. A. Haghighirad, D. V. Evtushinsky, M. Eschrig, and T. K. Kim, Scaling of the superconducting gap with orbital character in FeSe, Phys. Rev. B 98, 180503(R) (2018).

[24] Y. S. Kushnirenko, A. V. Fedorov, E. Haubold, S. Thirupathaiah, T. Wolf, S. Aswartham, I. Morozov, T. K. Kim, B. Büchner, and S. V. Borisenko, Three-dimensional 
superconducting gap in FeSe from angle-resolved photoemission spectroscopy, Phys. Rev. B 97, 180501(R) (2018).

[25] M. H. Christensen, R. M. Fernandes, and A. V. Chubukov, Orbital transmutation and the electronic spectrum of FeSe in the nematic phase, Phys. Rev. Research 2, 013015 (2020).

[26] L. Benfatto, B. Valenzuela, and L. Fanfarillo, Nematic pairing from orbital selective spin fluctuations in FeSe, npj Quantum Mater. 3, 56 (2018).

[27] A. Kreisel, B. M. Andersen, P. O. Sprau, A. Kostin, J. C. Séamus Davis, and P. J. Hirschfeld, Orbital selective pairing and gap structures of iron-based superconductors, Phys. Rev. B 95, 174504 (2017).

[28] H. Iwasawa, P. Dudin, K. Inui, T. Masui, T. K. Kim, C. Cacho, and $\mathrm{M}$. Hoesch, Buried double $\mathrm{CuO}$ chains in $\mathrm{YBa}_{2} \mathrm{Cu}_{4} \mathrm{O}_{8}$ uncovered by nano-ARPES, Phys. Rev. B 99, 140510(R) (2019).

[29] M. D. Watson, P. Dudin, L. C. Rhodes, D. V. Evtushinsky, H. Iwasawa, S. Aswartham, S. Wurmehl, B. Büchner, M. Hoesch, and T. K. Kim, Probing the reconstructed Fermi surface of antiferromagnetic $\mathrm{BaFe}_{2} \mathrm{As}_{2}$ in one domain, npj Quantum Mater. 4, 36 (2019).

[30] A. E. Böhmer, F. Hardy, F. Eilers, D. Ernst, P. Adelmann, P. Schweiss, T. Wolf, and C. Meingast, Lack of coupling between superconductivity and orthorhombic distortion in stoichiometric single-crystalline FeSe, Phys. Rev. B 87, 180505(R) (2013).

[31] B. Rösner, P. Dudin, J. Bosgra, M. Hoesch, and C. David, Zone plates for angle-resolved photoelectron spectroscopy providing sub-micrometre resolution in the extreme ultraviolet regime, J. Synchrotron Radiat. 26, 467 (2019).

[32] Most of the data were obtained with the analyzer set in fixed mode, and some artifacts due to the presence of the analyzer grid may be observed in the data, with the exception of the swept mode spectra in Figs. 3(c) and 3(f).

[33] D. Liu, C. Li, J. Huang, B. Lei, L. Wang, X. Wu, B. Shen, Q. Gao, Y. Zhang, X. Liu, Y. Hu, Y. Xu, A. Liang, J. Liu, P. Ai, L. Zhao, S. He, L. Yu, G. Liu, Y. Mao, X. Dong, X. Jia, F. Zhang, S. Zhang, F. Yang, Z. Wang, Q. Peng, Y. Shi, J. Hu, T. Xiang, X. Chen, Z. Xu, C. Chen, and X. J. Zhou, Orbital Origin of Extremely Anisotropic Superconducting Gap in Nematic Phase of FeSe Superconductor, Phys. Rev. X 8, 031033 (2018).

[34] It should be noted that our filtering procedure leads to a systematic bias towards finding intense holelike bands inside the ROI in Fig. 1(f); however, there is no systematic bias towards the absence of an electronlike pocket in Fig. 1(f) but its strong presence in Fig. 1(g), which further confirms that we indeed resolve the domain structure.

[35] L. C. Rhodes, M. D. Watson, A. A. Haghighirad, M. Eschrig, and T. K. Kim, Strongly enhanced temperature dependence of the chemical potential in FeSe, Phys. Rev. B 95, 195111 (2017).

[36] Y. Suzuki, T. Shimojima, T. Sonobe, A. Nakamura, M. Sakano, H. Tsuji, J. Omachi, K. Yoshioka, M. Kuwata-Gonokami, T. Watashige, R. Kobayashi, S. Kasahara, T. Shibauchi,
Y. Matsuda, Y. Yamakawa, H. Kontani, and K. Ishizaka, Momentum-dependent sign inversion of orbital order in superconducting FeSe, Phys. Rev. B 92, 205117 (2015).

[37] Here we access approximately the $Z$ point of the second Brillouin zone, where matrix elements for $d_{x z}$ and $d_{y z}$ are reversed compared with the first [48].

[38] T. Hashimoto, Y. Ota, H. Q. Yamamoto, Y. Suzuki, T. Shimojima, S. Watanabe, C. Chen, S. Kasahara, Y. Matsuda, T. Shibauchi, K. Okazaki, and S. Shin, Superconducting gap anisotropy sensitive to nematic domains in FeSe, Nat. Commun. 9, 282 (2018).

[39] E. F. Schwier, H. Takita, W. Mansur, A. Ino, M. Hoesch, M. D. Watson, A. A. Haghighirad, and K. Shimada, Applications for ultimate spatial resolution in LASER based $\mu$-ARPES: A FeSe case study, AIP Conf. Proc. 2054, 040017 (2019).

[40] T. Chen, Y. Chen, A. Kreisel, X. Lu, A. Schneidewind, Y. Qiu, J. T. Park, T. G. Perring, J. R. Stewart, H. Cao, R. Zhang, Y. Li, Y. Rong, B. M. Wei, Y. Andersen, P. J. Hirschfeld, C. Broholm, and P. Dai, Anisotropic spin fluctuations in detwinned FeSe, Nat. Mater. 18, 709 (2019).

[41] P. O. Sprau, A. Kostin, A. Kreisel, A. E. Böhmer, V. Taufour, P. C. Canfield, S. Mukherjee, P. J. Hirschfeld, B. M. Andersen, and J. C. S. Davis, Discovery of orbital-selective Cooper pairing in FeSe, Science 357, 75 (2017).

[42] L. C. Rhodes, M. D. Watson, T. K. Kim, and M. Eschrig, $k_{z}$ Selective Scattering within Quasiparticle Interference Measurements of FeSe, Phys. Rev. Lett. 123, 216404 (2019).

[43] F. Hardy, M. He, L. Wang, T. Wolf, P. Schweiss, M. Merz, M. Barth, P. Adelmann, R. Eder, A. A. Haghighirad, and C. Meingast, Calorimetric evidence of nodal gaps in the nematic superconductor FeSe, Phys. Rev. B 99, 035157 (2019).

[44] X Long, S. Zhang, F. Wang, and Z. Liu, Electronic nematicity in FeSe: a first-principles perspective, arXiv:1907.03562.

[45] P. V. Nguyen, N. C. Teutsch, N. P. Wilson, J. Kahn, X. Xia, A. J. Graham, V. Kandyba, A. Giampietri, A. Barinov, G. C. Constantinescu, N. Yeung, N. D. M. Hine, X. Xu, D. H. Cobden, and N. R. Wilson, Visualizing electrostatic gating effects in twodimensional heterostructures, Nature 572, 220 (2019).

[46] S. Ulstrup, C. E. Giusca, J. A. Miwa, C. E. Sanders, A. Browning, P. Dudin, C. Cacho, O. Kazakova, D. K. Gaskill, R. L. Myers-Ward, T. Zhang, M. Terrones, and P. Hofmann, Nanoscale mapping of quasiparticle band alignment, Nat. Comm. 10, 3283 (2019).

[47] M. Cattelan and N. A. Fox, A perspective on the application of spatially resolved ARPES for 2D materials, Nanomaterials $\mathbf{8}$, 284 (2018).

[48] V. Brouet, M. F. Jensen, P. H. Lin, A. Taleb-Ibrahimi, P. Le Fèvre, F. Bertran, C. H. Lin, W. Ku, A. Forget, and D. Colson, Impact of the two Fe unit cell on the electronic structure measured by ARPES in iron pnictides, Phys. Rev. B 86, 075123 (2012). 